Avoiding Resistance Limitations in High-Performance Transparent

Supercapacitor Electrodes Based on Large-Area, High-Conductivity

\title{
PEDOT:PSS Films
}

Thomas M Higgins and Jonathan N Coleman*

School of Physics, CRANN and AMBER research centres, Trinity College Dublin, Dublin 2,

Ireland

*colemaj@tcd.ie

\section{KEYWORDS:}

Printed electronics, transparent electronics, conducting polymer, PEDOT:PSS, electrochemical capacitor, supercapacitor, percolation.

ToC fig
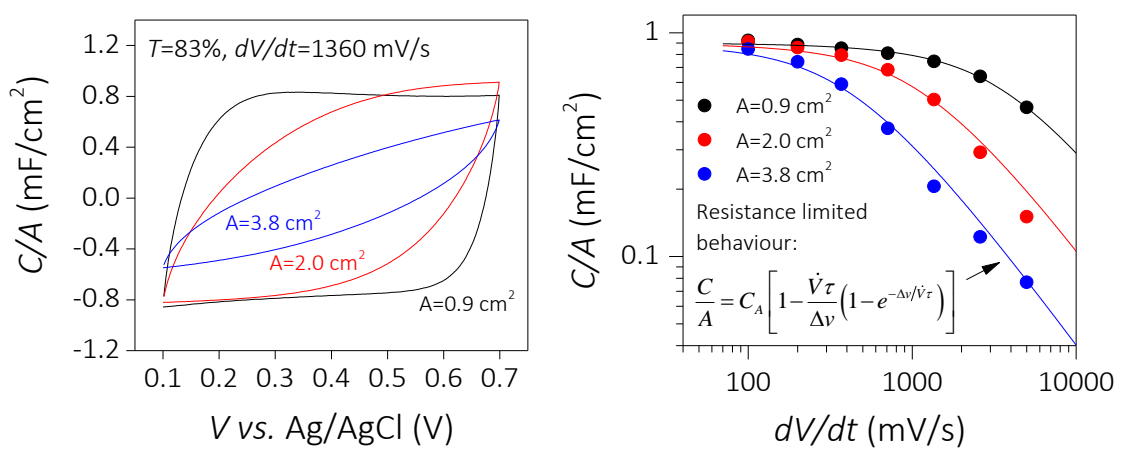


\section{ABSTRACT}

This work describes the potential of thin, spray-deposited, large-area poly(3,4ethylenedioxythiophene)/poly(styrene-4-sulfonate) (PEDOT:PSS) conducting polymer films for use as transparent supercapacitor electrodes. These films have a very low optical conductivity of $\sigma_{o p}=24 \mathrm{~S} / \mathrm{cm}$ (at $550 \mathrm{~nm}$ ), crucial for this application, and a reasonable volumetric capacitance of $C_{V}=41 \mathrm{~F} / \mathrm{cm}^{3}$. Secondary doping with formic acid gives these films a DC conductivity of $\sigma_{D C}=936 \mathrm{~S} / \mathrm{cm}$, allowing them to perform both as a transparent conductor/current collector and transparent supercapacitor electrode. Small area films $(A \sim 1$ $\mathrm{cm}^{2}$ ) display measured areal capacitance as high as $1 \mathrm{mF} / \mathrm{cm}^{2}$ even for reasonably transparent electrodes $(T \sim 80 \%)$. However, in real devices the absolute capacitance will be maximised by increasing the device area. As such, here we measure the electrode performance as a function of its length and width. We find that the measured areal capacitance falls dramatically with scan rate and sample length but is independent of width. We show that this is because the measured areal capacitance is limited by the electrical resistance of the electrode. We have derived an equation for the measured areal capacitance as a function of scan rate and electrode lateral dimensions which fits the data extremely well up to scan rates of $\sim 1000 \mathrm{mV} / \mathrm{s}$ (corresponding to charge/discharge times $>0.6 \mathrm{sec}$ ). These results are self-consistent with independent analysis of the electrical and impedance properties of the electrodes. These results can be used to find limiting combinations of electrode length and scan rate, beyond which electrode performance falls dramatically. We use these insights to build large area $\left(\sim 100 \mathrm{~cm}^{2}\right)$ supercapacitors using electrodes which are $95 \%$ transparent, providing a capacitances of $\sim 12$ $\mathrm{mF}$ (at $50 \mathrm{mV} / \mathrm{s}$ ), significantly higher than any previously reported transparent supercapacitor. 


\section{INTRODUCTION}

Recently, exciting progress has being made towards the creation of electronic devices on everyday plastic substrates by liquid phase processing techniques such as ink-jet and aerosol-jet printing. ${ }^{1-3}$ This approach is exciting as it will enable devices with unusual characteristics such as mechanically flexibility, stretchability and even transparency. ${ }^{3-5}$ It is likely that these possibilities will lead to numerous applications that are impossible using traditional manufacturing techniques.

In moving towards this goal, a wide range transparent electrical device components have already been demonstrated. Examples include conducting elements and circuitry, ${ }^{6-7}$ capacitors,${ }^{8}$ diodes,${ }^{9}$ transistors, ${ }^{10}$ audio speakers ${ }^{11}$ and sensors for both chemical ${ }^{12}$ and mechanical ${ }^{13}$ stimuli. In addition to these energy consuming components, transparent energy harvesting systems are also under investigation to impart power generation capabilities, for example, using photovoltaic ${ }^{14}$ and piezoelectric ${ }^{15}$ technologies.

To mediate between these energy harvesting and consuming components, transparent energy storage systems must also be developed. As for traditional electronics, this role will most likely be played by batteries and supercapacitors (SCs). Whilst examples of the former are presently scarce, ${ }^{16}$ significant efforts are underway to develop transparent/flexible SCs. ${ }^{17-}$ 35 The electrode materials under investigation for this purpose are numerous, including carbon nanotubes, ${ }^{27,} 32-33$ graphene, ${ }^{21,} 29,34$ transition metal oxides ${ }^{17,} 19-20,23,25$ and conducting polymers. ${ }^{17,22,30}$

In some transparent SC reports, the capacitive charge storage materials have been supported by an underlying transparent indium-tin oxide (ITO) layer to provide efficient current collection. ${ }^{19-25}$ However, due to its brittle nature, the growing cost of indium and need for both high-vacuum and high-temperature processing, ${ }^{36}$ it is unlikely this material will be 
compatible with future printed electronics. Rather, we believe it is more likely that future transparent/flexible SCs will contain ITO-free electrodes deposited directly on plastic substrates. Thus, developing an electrode material that can simultaneously provide excellent capacitive charge storage, efficient current collection and high optical transparency is in important problem in this field.

This being the case, here we briefly outline some key physical relations and figures of merit relevant for characterising the performance of transparent SC electrodes. As for any SC electrode, the primary measures of performance are the stored energy $(E)$ and power output $(P)$, given by:

$E=\frac{1}{2} C \Delta V^{2}$

$$
P=\frac{1}{4} \frac{\Delta V^{2}}{R_{E S R}}
$$

While the electrode interfacial potential difference, $\Delta V$, is clearly important, it is strongly influenced by the properties of the electrolyte $(\sim 1.2 \mathrm{~V}$ for devices that employ aqueous electrolytes). Most important from a materials standpoint are the electrode capacitance, $C$, and associated equivalent series resistance, $R_{E S R}$, which must be maximised and minimised, respectively. For this specific application it is crucial to understand how both of these properties are related to the electrode transparency, $T$.

The capacitance a given electrode is able to supply depends its volumetric capacitance, $C_{V}$, and its dimensions. In practical terms, the areal capacitance, $C_{A}$, is the most relevant measure of capacitance in transparent/flexible devices considering that multiple device components must be assembled onto a given area of substrate (e.g. handheld devices, functional 
packaging or e-labels). The areal and volumetric capacitance are related $v i a C_{A}=C_{V} t$, where $t$ is the film thickness. As defined here, $C_{A}$ and $C_{V}$ are both rate-independent quantities and hence indicate the capacitance realised in the absence of charge transport limitations (these limitations will be discussed later). The $C_{A}$ of a transparent electrode is related to its optical transmittance, $T$, via the expression: ${ }^{27}$

$T=\left(1+\frac{Z_{0} \sigma_{o p}}{2 C_{V}} C_{A}\right)^{-2}$

where $\mathrm{Z}_{0}$ is a constant $(377 \mathrm{Ohm})$ and $\sigma_{o p}$ is the optical conductivity. This property is a measure of how much light the electrode material absorbs and it is directly proportional to the Beer-Lambert absorption coefficient. Clearly the ratio $C_{V} / \sigma_{o p}$ must be maximised to optimise the areal capacitance available for a given electrode transparency, and thus it can be considered a figure of merit for transparent capacitive films $\left(\mathrm{FoM}_{\mathrm{c}}\right)$. We emphasise that unlike traditional $\mathrm{SC}$ electrodes, the gravimetric capacitance, $\mathrm{C}_{\mathrm{M}}$, is of little relevance when characterising transparent SCs electrodes. This is because the mass of the electrode materials are insignificant compared with the plastic substrates supporting them. In addition, it is difficult to measure the mass of ultra-thin films and therefore use of this metric inhibits the accurate comparison of different transparent supercapacitor materials.

Regarding the electrode equivalent series resistance, it is known that the $R_{E S R}$ of transparent charge storage devices depends strongly on the electrical properties of the charge storage films, which in this situation must also act as current collectors. ${ }^{27}$ To characterise this behaviour the electrode sheet resistance, $R_{s}$, can be related to $T$ via a similar expression as for the areal capacitance: ${ }^{37}$ 
$T=\left(1+\frac{Z_{0}}{2 R_{s}} \frac{\sigma_{o p}}{\sigma_{D C}}\right)^{-2}$

Here $\sigma_{D C}$ is the electrical conductivity of the thin film material. Thus, here the ratio $\sigma_{D C} / \sigma_{o p}$ provides an electrical figure of merit $\left(\mathrm{FoM}_{\mathrm{e}}\right)$ that must be maximised when optimising the power output capabilities of transparent charge storage films.

In summary, although a thin film can have any desired transparency by making it sufficiently thin, doing so entails a corresponding increase in $R_{s}$, with implications for current collection and hence power. Moreover, high $T$ simultaneously results in reduced $C_{A}$, hence a reduction in the energy which can be stored by a device with a given footprint. ${ }^{27,32}$ Quantifying these trade-offs using the described figures of merit will greatly assist with the development of high performance transparent SCs.

In addition to these considerations, in this work we identify a further property trade-off that is important for the design of transparent SCs: For a given electrode material (with some $\sigma_{D C}, \sigma_{o p}$ and $C_{V}$ ), if the film thickness is constrained to maintain some desired transparency, the only remaining option to attain larger absolute capacitance is by increasing the lateral dimensions of the electrodes. In doing so, $C$ should scale linearly with film area (i.e. $C=C_{A} A$ ), especially for smaller electrodes that are charged/discharged slowly. However, for larger films, eventually charge storage sites more distant from the external electrical contact will become inaccessible, particularly when the film is charged and discharged quickly. Understanding such lateral dimension effects will be necessary for scaling-up device area for application in transparent electronics. 
Here we address this need by exploring the transparent SC properties of a sprayed conducting polymer, PEDOT:PSS, as a function of both film thickness and lateral dimensions. This commercially available conducting polymer has shown much potential to replace ITO within low-cost printed electronics applications such as organic solar cells ${ }^{38-40}$ and organic light emitting diodes. ${ }^{40}$ In addition, it is also being explored for use within both SC and battery electrodes. ${ }^{41}$ As such, a detailed exploration of the potential of sprayed PEDOT:PSS for use within transparent/flexible energy storage applications is required. 


\section{RESULTS \& DISCUSSION}

\section{Optoelectronic Properties of Sprayed PEDOT:PSS Films}

Aqueous solutions of PEDOT:PSS were deposited onto flexible plastic substrates (PET) to form thin films using a robot-controlled airbrush spray system. Figure 1A presents a photograph of a characteristically blue aqueous PEDOT:PSS solution and a typical film produced $(T=70 \%)$. Although relatively small area films were prepared for initial characterisation $(5 \mathrm{~cm} \times 5 \mathrm{~cm})$, this spraying system allows the production of films up to 18 $\mathrm{cm} \times 18 \mathrm{~cm}$ (SI Figure S1). In principle, the stage area could be extended to produce arbitrarily large films. Details of the spray parameters employed in this work are given in the SI.

The film transmittance, $T$, was varied by altering the volume of PEDOT:PSS solution deposited onto the substrate. This was quantified by measuring UV-visible transmittance spectra, presented in Figure 1B. For conducting thin films, the transmittance for a given thickness, $t$, is controlled by the optical conductivity of the material, $\sigma_{o p}$, via the Equation: ${ }^{42}$

$T=\left[1+Z_{0} \sigma_{o p} t / 2\right]^{-2}$

Although use of this expression isn't common within the transparent SC field, it is frequently employed by transparent conductor researchers. ${ }^{37}$ We note that by expansion to first order, Equation 5 is identical to the Lambert-Beer law $\left(T=e^{-\alpha t}\right)$ with $Z_{0} \sigma_{o p} \approx \alpha$, the optical absorption coefficient. The optical conductivity of PEDOT:PSS was determined by measuring the thickness of a number of sprayed films using profilometry, which found values between $470 \mathrm{~nm}$ and $1600 \mathrm{~nm}$. (N.B. these measurements were obtained after the formic acid secondary doping treatment described below for films that were sprayed onto glass substrates). Plotting 
this data as $T^{-1 / 2}-1$ vs. $t(550 \mathrm{~nm}$, Figure 1C) and fitting Equation 5, we obtained a value of $\sigma_{o p}=24 \mathrm{~S} / \mathrm{cm}$ (at $550 \mathrm{~nm}$ ) for these PEDOT:PSS films. For comparison, data for spin-coated PEDOT:PSS films described by Martin et al. ${ }^{43}$ is included (unfilled points), falling close to our fitted curve. This value for $\sigma_{o p}$ is extremely low compared to other conducting materials: silver nanowires display values in the range $38-65 \mathrm{~S} / \mathrm{cm},{ }^{44-45}$ SWNTs have $150-200 \mathrm{~S} / \mathrm{cm},{ }^{46-47}$ graphene has $100-2000 \mathrm{~S} / \mathrm{cm}^{48}$ We believe this low optical conductivity of PEDOT:PSS will be critical to its success within transparent SCs (or conductors). Using $\sigma_{o p}=24 \mathrm{~S} / \mathrm{cm}$ with Equation 5, the thickness of any PEDOT:PSS film can be determined from its transmittance spectrum. This is useful as it avoids difficulties associated with obtaining accurate thickness measurements of ultra-thin films by physical methods such as atomic force microscopy. For the spectra shown in Figure 1B, $t$ varied between 17 and $432 \mathrm{~nm}$.

As for traditional SC electrodes, electronic transport to and from the electrochemical interface must occur efficiently to enable high power device operation. ${ }^{27,49}$ Accordingly, next we characterised the electrical properties of these PEDOT:PSS films to evaluate their suitability to act as transparent electrodes without additional current collectors. Although the electrical conductivity of as-prepared PEDOT:PSS films are relatively poor, dramatic improvements are easily obtained by exposing the films to a variety of 'secondary-dopants' such as sorbitol, ${ }^{50}$ ethylene glycol, ${ }^{36}$ dimethyl sulfoxide ${ }^{36}$, methanol ${ }^{51}$ and $\mathrm{H}_{2} \mathrm{SO}_{4}{ }^{52}$. Here we have used the simple treatment reported by McCarthy et al., ${ }^{53}$ which involves dipping the PEDOT:PSS films in formic acid (FA) at room temperature for $\sim 5$ seconds.

The effect of this treatment was evaluated using the four point probe method. We calculated the $R_{s}$ of these films before and after exposure to FA, which is plotted as a function of film transmittance in Figure 1D. Clearly, the treatment dramatically improved the electrical 
properties of these transparent films, consistent with previous reports. ${ }^{53}$ At high transmittance (e.g. $T=90 \%$ ), the sheet resistance is reduced by 3 orders of magnitude, falling from an untreated value of $R_{s}=1.5 \times 10^{5} \mathrm{Ohm} / \mathrm{sq}$ to $102 \mathrm{Ohm} / \mathrm{sq}$ after treatment. We note that the film with $T=90 \%$ and $R_{s}=102 \mathrm{Ohm} / \mathrm{sq}$ represents exceptional performance for a transparent electrode of any sort. ${ }^{37}$ Only silver nanowire networks routinely display better performance. ${ }^{37}$, ${ }^{44}$ We can determine the ratio of DC to optical conductivities as a figure of merit $\left(\mathrm{FoM}_{\mathrm{e}}\right)$ for transparent conductors by fitting Equation 4 to the data in Figure 1D. For these PEDOT:PSS films, we find a value of $\sigma_{D C} / \sigma_{o p}=39$. For comparison, data for transparent current collectors formed by disordered networks of two different types of SWNTs are also shown; those characterised as transparent supercapacitors by King et al., ${ }^{27}$ and Iljin SWNTs characterised in this work (see SI for SWNT network preparation). Fitting Equation 4 to these data sets yields $\sigma_{D C} / \sigma_{o p}=2$ and 5, respectively, highlighting the excellent performance of these FA-treated PEDOT:PSS films. We note that the transmittance spectra were largely unchanged by the FA post-treatment (SI Figure S2A), indicating that increased $\sigma_{D C}$ is responsible for the improved FoMe rather than due to substantial changes in $\sigma_{o p}$.

As the optical conductivity is known, we can calculate the DC conductivity of the treated PEDOT:PSS films using the FoMe as $\sigma_{D C}=936 \mathrm{~S} / \mathrm{cm}$. This value is quite high for polymeric conductors, albeit not as high as some other recent reports; PEDOT:PSS films have been demonstrated with a DC conductivity of $1418 \mathrm{~S} / \mathrm{cm}$ when treated with ethylene glycol ${ }^{36}$ and $3000 \mathrm{~S} / \mathrm{cm}$ when treated with $1.5 \mathrm{M} \mathrm{H}_{2} \mathrm{SO}_{4}$ at $160^{\circ} \mathrm{C} .{ }^{52}$ This value is competitive with the best data for carbon nanotube films ${ }^{37}$ and significantly better than most solution-processed graphene electrodes ${ }^{54}$ Although, a metal grid or mesh could be deposited on the film to further improve current collection this would impair the electrode transmittance and complicate electrode processing. Rather, it would be preferrable that an single electrode material could be 
used to provide sufficient electrical conductivity and high capacitive charge storage simultaneously.

Considering Figure 1D, for both the SWNT and untreated PEDOT:PSS data, there is marked deviation from the fits described by Equation 4 at higher transmittance values. These deviations are more apparent by replotting the data as $T^{-1 / 2}-1$ vs. $R_{s}$, shown in SI Figure S2B. Here the linearized fits correspond to bulk-like behaviour. Deviations from Equation 4 have been explained as a manifestation of percolation-type phenomena. ${ }^{55}$ While this is probably the case for the nanotube films, for the untreated PEDOT:PSS deviation from bulk-like behaviour is probably due to the presence of islands of PEDOT rich grains interspersed within a network insulating PSS barriers. ${ }^{56}$ Importantly, no such deviations in optoelectronic properties are observed for the FA-treated PEDOT:PSS films, indicating exceptional uniformity even for films with $T=99 \%$. This is significant as such deviations always diminish the performance of transparent SCs at high $T .{ }^{27}$ Networks formed from typical-sized discrete nano-objects such as carbon nanotubes, graphene or metallic wires almost always show percolation effects as film thickness is reduced. ${ }^{37}$

The superlative optical and electrical properties of secondary-doped PEDOT:PSS films make this material ideal for the production of transparent SC electrodes. The low optical conductivity means that for a given transmittance (e.g. $T=95 \%$ ) the electrode can be relatively thick. For a given intrinsic volumetric capacitance this will result in a larger areal capacitance $\left(C_{A}=C_{V} t\right)$. Additionally, high DC conductivity will facilitate transport of stored charge to the external circuit even for electrodes with high $T$. 
The capacitive charge storage properties of small area PEDOT:PSS films were characterised in a three electrode cell by cyclic voltammetry (CV). This allows characterisation of the properties of the electrode material itself rather than a complete SC device. We first carried out $\mathrm{CV}$ measurements for a $T=95 \%$ film over a wide potential window $(-0.8$ to $1.0 \mathrm{~V}$ vs $\mathrm{Ag} / \mathrm{AgCl}$ ), shown in SI Figure S3. Based on this we chose a narrower potential window for all subsequent electrochemical measurements in a region where the electrode behaviour was ideally capacitive (-0.1 to $0.7 \mathrm{~V}$ vs. $\mathrm{Ag} / \mathrm{AgCl})$. Although future studies aimed at maximising energy storage could focus on maximising the potential window (Equation 1), here we do not focus on this aspect of SC electrode performance.

Shown in Figure 2A are CV scans for a PEDOT:PSS film $(T=95 \%, \dot{V}=100 \mathrm{mV} / \mathrm{s})$ before and after exposure to formic acid. A series resistor and capacitor circuit provides a simple model for the electrode current response to a linear potential sweep, expressed as a function of time, $t:^{57}$

$$
j=C_{A} \dot{V}\left(1-e^{-t / \tau}\right)
$$

Here $j$ is the current density, $\dot{V}=d V / d t$ is the scan rate, $C_{A}$ is the rate-independent electrode areal capacitance already introduced and $\tau=R_{E S R} C$ is the time constant. Here $R_{E S R}$ is the equivalent series resistance of the electrochemical circuit (here in a three-electrode configuration) and $C=C_{A} A$ is the capacitance of the electrode. The rapid current response of the FA-treated film compared with the as-prepared film indicates a dramatic reduction in $\tau$. We believe this improved electrode response is overwhelmingly due to improved current 
collection through the plane of the film, as the treatment caused the $R_{s}$ of this film to decrease by over two orders of magnitude, giving $205 \mathrm{Ohm} / \mathrm{sq}$ compared with $100 \mathrm{kOhm} / \mathrm{sq}$ prior to the treatment. This view is also supported by the previous observation that the $R_{E S R}$ of transparent SC electrodes scales linearly with the $R_{s}{ }^{27}$ In addition, alternative explanations for the large reduction in $\tau$ are unlikely, such as due to reduced electrode capacitance or other contributions to the $R_{E S R}$ such as the electrolyte or electrode contact resistance.

To assess the electrochemical stability of the FA-treated films, 3500 repeated CV cycles were performed for both thin and thick samples ( $T=67 \%$ and $T=95 \%)$ at a scan rate of 100 $\mathrm{mV} / \mathrm{s}$. By integrating the current passed per cycle, a capacitance retention ratio was calculated and plotted as a function of the number of CV cycles in Figure 2B. We observed a slight increase in capacitance within the first 1000 cycles $(<3 \%$ for the thicker film and $<1 \%$ for the thin film) probably due to minor rearrangement of the polymer film. With $>99 \%$ retention for both films, the charge/discharge process is stable over this duration of cycling. The Figure $2 \mathrm{~B}$ inset compares $\mathrm{CV}$ trace of the 1 st and 3500 th cycle for the $T=95 \%$ film, indicating some minor changes in features of the CV. Alongside the well-known stability of PEDOT, ${ }^{41,58}$ these results suggest excellent cyclability of this material, a prerequisite of any promising SC electrode.

We then examined the charge storage properties of the small area $(1 \mathrm{~cm} \times 1 \mathrm{~cm}) \mathrm{FA}$ treated PEDOT:PSS films in more detail. Here CV measurements were obtained at a range of scan rates $(\dot{V}=100-5000 \mathrm{mV} / \mathrm{s})$ and the current density at mid-voltage $(0.4 \mathrm{~V} v s . \mathrm{Ag} / \mathrm{AgCl})$ was evaluated. In the ideal case, capacitive behaviour is indicated by $j=C_{A} \dot{V}{ }^{59}$ We note that Equation 6 reduces to this form when the electrode time constant is small relative to the discharge or recharging time (i.e. $\tau<<\Delta V / \dot{V}$ ). Figure $2 \mathrm{C}$ shows such data obtained from $\mathrm{CV}$ 
measurements of films with different transmittance ( $T=80 \%$ to $99 \%, t=10-260 \mathrm{~nm}$ ). The inset shows example CV traces for these films at $100 \mathrm{mV} / \mathrm{s}$. Clearly, near ideal capacitive behaviour predominates over a wide range of scan rates for all of these small area films, even those at very high $T$. Similar behaviour was observed for PEDOT:PSS based SCs without an underlying current collector by Carlberg et al. which remain ideally capacitive at $200 \mathrm{mV} / \mathrm{s} .{ }^{41}$ For our films, only above $\sim 1000 \mathrm{mV} / \mathrm{s}$ do deviations become noticeable, corresponding to charge and discharge times less than $\sim 0.6$ seconds. Closer inspection reveals that for thicker films (with lower $R_{s}$ ), the onset of this deviation shifts to greater scan rates. This provides a subtle indication that the transport limitations which determine $R_{E S R}$ are likely due to the $R_{s}$ contributions as opposed to ionic diffusion, for which we would expect the deviation onset to be independent of film transmittance.

Considering the data in Figure 2C, using the low scan rate range over which the electrode behaviour is near ideally capacitive $(\dot{V}<1000 \mathrm{mV} / \mathrm{s})$, we extracted the rateindependent quantity, $C_{A}$, for each film from the intercept with the ordinate. This data has been plotted a function of film transmittance in Figure 2D. As expected, more transparent films store less charge for a given geometric area, simply because thinner films provide less capacitive material on the substrate surface. We measured $C_{A}=1.9 \mathrm{mF} / \mathrm{cm}^{2}$ for a low transmittance film ( $T=70 \%)$, decreasing to $C_{A}=0.18 \mathrm{mF} / \mathrm{cm}^{2}$ for the most transparent film $(T=99 \%)$. To the best of our knowledge the areal capacitance values of these FA treated PEDOT:PSS films sets the state of the art for high SC electrodes with high transmittance (T>90\%).

Provided $C_{V}$ is indeed constant for all film thicknesses, these data should be described by Equation 3. ${ }^{27}$ This equation describes the data well for all transmittances. Because this is equivalent to showing $C_{A} \propto t$ for all thicknesses, ${ }^{27}$ this indicates that the electrolyte penetrates 
throughout the PEDOT:PSS films. Fitting gives $C_{V} / \sigma_{o p}=1.7$ F/S.cm ${ }^{2}$, which can be considered a figure of merit for transparent capacitive electrodes $\left(\mathrm{FoM}_{\mathrm{c}}\right)$. Alongside these PEDOT:PSS data we have also included results from the transparent capacitor literature. ${ }^{8,17,22,}$ 26, 28-32, 34 Note that this excludes reports where underlying ITO current collectors have been used. ${ }^{18-25}$ We highlight that other than this work and that of King et al.,${ }^{27}$ to our knowledge no other data has been presented for electrodes with $T>90 \%$, the region of interest for transparent devices. SI Figure S4 contains a reproduction of Figure 2D indicating the authors of the literature data, which weren't included for clarity. Our PEDOT:PSS films have a much higher FoMc than the disordered SWNT films reported by King et al., which provide $C_{V} / \sigma_{o p}=0.3$ $\mathrm{F} / \mathrm{S} . \mathrm{cm}^{2}$. In addition, while the capacitance of the SWNT network films deviate markedly from bulk-like behaviour at $T>90 \%$ due to percolation effects, ${ }^{27}$ the capacitance of these PEDOT:PSS films remain bulk-like even at very high transmittance $(T=99 \%)$. As discussed above, this is a key advantage of these sprayed PEDOT:PSS films compared alternative transparent SC electrodes based on disordered nanomaterial network electrodes.

Equation 3 indicates that the capacitive performance of transparent SC electrodes depends strongly on $C_{V}$. However, this quantity is rarely calculated explicitly for transparent SC electrodes. We can determine the volumetric capacitance of this material using the fitted FoMc and the known $\sigma_{o p}$ of this material, yielding $C_{V}=41 \mathrm{~F} / \mathrm{cm}^{3}$. Despite the disparity between the FoMc of these PEDOT:PSS films $\left(C_{V} / \sigma_{o p}=1.7 \mathrm{~F} / \mathrm{S} . \mathrm{cm}^{2}\right)$ and the SWNT films reported by King et al. $\left(C_{V} / \sigma_{o p}=0.3 \mathrm{~F} / \mathrm{S} . \mathrm{cm}^{2}\right)$, it's interesting to note that these two materials have similar volumetric capacitances ( $C_{V}=55 \mathrm{~F} / \mathrm{cm}^{3}$ for the SWNTs). Therefore, again we can attribute the superior performance of PEDOT:PSS as a transparent SC charge storage film to its very low optical conductivity $\left(\sigma_{o p}=24 \mathrm{~S} / \mathrm{cm}\right.$ compared with $\sigma_{o p}=167 \mathrm{~S} / \mathrm{cm}$ for the 
SWNTs). Using this value for the volumetric capacitance we can calculate an approximate gravimetric capacitance of this materials using $C_{M}=C_{V} / \rho$. Here we have measured density of a free-standing PEODT:PSS film as $\rho=1.2 \mathrm{~g} / \mathrm{cm}^{3}$, giving $C_{M}=34 \mathrm{~F} / \mathrm{g}$, a fairly typical value for an electrochemical double layer capacitance. Although PEDOT is capable of pseudocapacitive charge storage through reduction at potenials $\sim 0.2 \mathrm{~V}$ vs. $\mathrm{Ag} / \mathrm{AgCl}$ and subsequent reoxidisation at $\sim 0.0 \mathrm{~V}$ (see SI Figure 3) the use of such processes may not be suitable for transparent SC applications due to the associated electrochromism. ${ }^{40}$

Many electrode materials have been reported with substantially higher volumetric capacitance, particularly those making use of pseudocapacitive charge transfer reactions; Carlberg et al. reported $100 \mathrm{~F} / \mathrm{cm}^{3}$ for PEDOT:PSS films, ${ }^{41}$ Ghaffari et al. measured $84 \mathrm{~F} / \mathrm{cm}^{3}$ for PEDOT:PSS coated nanotubes, Largeot et al. ${ }^{60}$ reported values $\sim 100 \mathrm{~F} / \mathrm{cm}^{3}$ for nanotubes, and values as high as $1160 \mathrm{~F} / \mathrm{cm}^{3}$ have been achieved using $\mathrm{MnO}_{2}$ composites. ${ }^{61}$ The literature data in Figure 2D which sits ahead of our PEDOT:PSS data in the low transmittance regime (Gao et al. ${ }^{28}$ and Nam et al. ${ }^{31}$ ) suggests that higher FoMc than that of these PEDOT:PSS films are possible (i.e. $C_{V} / \sigma_{o p}$ as high as $5 \mathrm{~F} / \mathrm{S} . \mathrm{cm}^{2}$ ). These electrode materials could yield better capacitance at high transmittance values $(T>90 \%)$ if they could be produced thin enough while also avoiding percolation effects. ${ }^{27}$ We suggest this may be achieved by composite formation of secondary-doped PEDOT:PSS with another highly pseudocapacitive material such as manganese dioxide ${ }^{62}$ to boost $C_{V}$ without substantially increasing $\sigma_{o p}$.

Considering Figure $2 \mathrm{C}$, when the scan rate is large enough, the measured areal capacitance (which we will refer to as $C / A$ and represents the accessible area capacitance) becomes less than the areal capacitance expected in the absence of electronic or ionic transport limitations (i.e. $C / A<C_{A}$ ). We can examine this scan rate dependence of the $\mathrm{CV}$ behaviour 
more effectively by normalising the measured current density to the scan rate, yielding a differential capacitance (i.e. $j / \dot{V}=(d q / d V) / A) .{ }^{63}$ Figure 2E shows $\mathrm{CV}$ data expressed in this manner for a film with $T=99 \%$ at a variety of scan rates ( $\dot{V}=100$ to $5000 \mathrm{mV} / \mathrm{s}$ ). These demonstrate a reduction in accessible charge storage with scan rate due to the electrode time constant becoming comparable to the time available to accumulate or dispense charge. Although Figure 2C allowed extraction of this rate-independent areal capacitance, the measured areal capacitance, $C / A$, at a given scan rate is given by the total charge $(q)$ passed divided by the voltage window (i.e. $C / A=(\Delta q / \Delta V) / A$ ). This value can be found at each scan rate by integrating $\mathrm{CV}$ data using:

$$
\frac{C}{A}=\frac{1}{(\Delta V) \dot{V}} \int_{v}^{v+\Delta V} j d V
$$

Here $v$ is the starting voltage $(0.1 \mathrm{~V} v s . \mathrm{Ag} / \mathrm{AgCl})$ and $\Delta V$ is the potential window (here 0.6 V). The $C / A$ is shown in Figure $2 \mathrm{~F}$ for a number of films with different transmittance $(T=80 \%$ to $99 \%$ ). The included dashed lines are the rate-independent $C_{A}$ values obtained in Figure 2C. As expected, the $C / A$ values approach $C_{A}$ at low scan rates. The ratio of these quantities ( $\left.(C / A) / C_{A}\right)$ provides a measure of the capacitance that is accessible at a given scan rate. For example, for the $T=99 \%$ film, $92 \%$ of $C_{A}$ is accessible at $300 \mathrm{mV} / \mathrm{s}$ while this decreases to $75 \%$ at $1000 \mathrm{mV} / \mathrm{s}$. Although these deviations aren't dramatic, it's important to note that these films are relatively small $(1 \mathrm{~cm}$ by $1 \mathrm{~cm})$. Given that real transparent $\mathrm{SC}$ devices are likely to use electrodes with larger dimensions than this, it is necessary to also characterise the charge storage behaviour transparent films as a function of film size. 
For a charge storage material with some value of $C_{V}$, when film thickness is constrained to provide some required transmittance (say $T=90 \%$ ), the only way to store more charge is by increasing the film volume via the lateral dimensions of the film. However, in the literature the vast majority of transparent SC electrodes are tested with small active areas and obtained capacitance data presented on either a per mass or per area basis. In general, no attempt is made to explore the scaling of absolute capacitance with electrode dimensions for larger area electrodes.

Here we perform such a study, exploring the effect of both electrode length and width on the absolute capacitance. We define the electrode width, $W$, as the dimension parallel to the current collecting electrical contact at the upper film edge, and electrode length, $L$, as the perpendicular dimension (see Figure 3A. N.B we do not use a current collector between the PEDOT:PSS film and the PET support). Initially, we measured CVs for electrodes as a function of film $L$ (and so area) while keeping the width constant $(W=0.5 \mathrm{~cm})$. This was achieved by first characterising the largest area film, then cutting off a small bottom section from the film to reduce $L$. For example, Figures $3 \mathrm{~B}$ and $\mathrm{C}$ show the area dependence of the $\mathrm{CV}$ response of these films at $100 \mathrm{mV} / \mathrm{s}$ and $1000 \mathrm{mV} / \mathrm{s}$, respectively. Here $L$ was varied from $3.6 \mathrm{~cm}$ to 1.2 $\mathrm{cm}$, resulting in a variation in film area from $A=1.8 \mathrm{~cm}^{2}$ to $0.6 \mathrm{~cm}^{2}$. Again, the current density has been normalised to scan rate to enable its expression as a differential areal capacitance ( $(d q / d V) / A)$.

These results are interesting as they highlight the effect of both $L$ and $\dot{V}$ on the measured areal capacitance. At the relatively slow scan rate of $100 \mathrm{mV} / \mathrm{s}$, there is a small but noticeable increase in time constant as the sample length is increased, consistent with an 
increasing in-plane electrode resistance contributing to the $R_{E S R}$ and a larger available capacitance, which together determine the electrode time constant. This will manifest itself as a slight reduction in $C / A$ at a given $\dot{V}$. However, at the higher scan rate of $1000 \mathrm{mV} / \mathrm{s}$ this effect is much more pronounced. As $L$ is increased, significant increases in the time constant can be seen. These results imply that long electrodes will sustain reduced $C$ / $A$ compared to that expected for short ones, especially at high scan rates. This illustrates that the absolute capacitance of a potential device cannot simply be projected from the areal capacitance values measured for small area samples.

We can quantify this behaviour by calculating $C / A$ from the $\mathrm{CV}$ scans by integration (Equation 7). This has been performed for samples with a range of $L$ (with $W=1.0 \mathrm{~cm}$ ) with each one analysed over a range of $\dot{V}$, shown in Figure 4A. It is clear from this data that the capacitance falls dramatically as both $L$ and $\dot{V}$ are increased, as suggested above. These effects can be significant; at $\dot{V}=1000 \mathrm{mV} / \mathrm{s}$, and when $L=3.6 \mathrm{~cm}, C / A$ is a factor of three less than expected from the $L=1.2 \mathrm{~cm}$ sample, leading to no increase in absolute capacitance as the electrode area is increased.

The decrease in $C / A$ with increasing $L$ can be understood as follows. As the electrode gets longer, the average resistance felt by electrons moving from the electrode contact through the film increases. This in turn increases the time constant $\left(\tau=R_{E S R} C\right)$, causing a slower current rise on voltage reversal. Because the capacitance is calculated by integration of the $\mathrm{CV}$ curve, this results in lower measured areal capacitances that expected in the absence of charge transport limitations (equivalent to $C / A<C_{A}$ ). We can model this quantitatively by combining Equations 6 and 7 to give: 
$\frac{C}{A}=\frac{1}{\Delta V} \int_{v}^{v+\Delta V}\left[C_{A}\left(1-e^{-t / \tau}\right)\right] d V$

All the terms appearing have been described earlier. Changing variable from $V$ to $t$ ( $d V=\dot{V} d t$ ) and integrating gives:

$$
\frac{C}{A}=C_{A}\left[1-\frac{\dot{V} \tau}{\Delta V}\left(1-e^{-\Delta V / \dot{V} \tau}\right)\right]
$$

This describes the dependence of the measured areal capacitance, $C / A$, on the rateindependent areal capacitance, $C_{A}$, the scan rate, $\dot{V}$ and the time constant, $\tau$.

We can use Equation 9 to fit the $C / A$ vs. $\dot{V}$ data shown in Figure 4A, finding very good agreement for all samples lengths. From the fits we find that for this particular thickness of PEDOT:PSS ( $T=70 \%, t=432 \mathrm{~nm}$ ) the rate-independent areal capacitance is $C_{A}=1.9 \mathrm{mF} / \mathrm{cm}^{2}$. Note, we expect a value of $1.8 \mathrm{mF} / \mathrm{cm}^{2}$ for a film with this transmittance based on the $\mathrm{FoM}_{\mathrm{c}}$ (Figure 2D). It is worth noting that by expanding the exponential in Equation 9, it can be shown that at high scan rates, $C / A \propto \dot{V}^{-1}$ in the situation where the capacitance is limited by the electrical properties of the electrode. This is in comparison to the more well-known scenario where the measured area capacitance at high rates is limited by ionic diffusion and $C / A \propto \dot{V}^{-1 / 2}$ .${ }^{57}$ This allows the potential for rapid assessment of the limiting factor from the capacitance versus scan rate data.

In addition, we find that the time constant (from the fit) varies considerably with sample length, as shown in the Figure 4B. We can understand this by noting that the time constant is given by $\tau=R_{E S R} C$, where $C$ reflects the available capacitance of the electrode and $R_{E S R}$ 
reflects the series combination of electrode and electrolyte resistances associated with this electrode $\left(R_{E S R}=R_{\text {electrode }}+R_{\text {electrolyte }}\right)$. Here we assume that all the resistance associated with electrical contacts and the external leads are small enough to be ignored. We can express these three parameters in terms of the electrode geometry; the capacitance as $\left(C=C_{A} L W\right)$, the electrolyte resistance as the inverse of the electrolyte conductance per unit area, $G_{A}$, multiplied by area, $1 / G_{A} L W$ and the electrode resistance as the sheet resistance multiplied by electrode aspect ratio, $R_{s} L / W$. When combined, these given an expression for the time constant which is a function of electrode length, $L$, as shown in Equation 10.

$$
\tau=C\left(R_{\text {electrode }}+R_{\text {electrolyte }}\right)=C_{A} L W\left(R_{s} \frac{L}{W}+\frac{1}{G_{A} L W}\right)=C_{A} R_{s} L^{2}+\frac{C_{A}}{G_{A}}
$$

As shown in Figure 4B, Equation 10 fits the experimental data extremely well allowing extraction values of $R_{s}=24 \mathrm{Ohm} / \mathrm{sq}$ and $G_{A}=18 \mathrm{mS} / \mathrm{cm}^{2}$ for this sample. This film had a sheet resistance of $25 \mathrm{Ohm} / \mathrm{sq}$. when measured by current-voltage curves. This length dependence analysis has been repeated for a number of films with differing transmittance values ( $T=70$ to 90\%, shown in SI Figure S5). The extracted $R_{s}$ values have been plotted alongside the $R_{s}$ data obtained by current-voltage measurements in Figure 4D, showing excellent agreement. Shown in the inset of this figure are the $G_{A}$ values that were obtained. Although the true electrolyte conductance should be invariant of film thickness, the values obtained here differ slightly due to the fact that this parameter includes other resistance terms ignored for this analysis, in particular, the through film resistance between the electrode and electrical contact (shown in orange in Figure $3 \mathrm{~A})$. Given that $G_{A}=l \sigma_{\text {electrolyte }}$, where $\sigma_{\text {electrolyte }}$ is the electrolyte conductivity and $l$ is the electrode separation (here $\sim 1 \mathrm{~cm}$ ), this suggests that $\sigma_{\text {electrolyte }} \sim 15 \mathrm{mS} / \mathrm{cm}$, which 
is within a factor of five of the expected value for a $0.5 \mathrm{M} \mathrm{K}_{2} \mathrm{SO}_{4}$ solution $(75 \mathrm{mS} / \mathrm{cm}$ at 20 $\left.{ }^{\circ} \mathrm{C}\right) .{ }^{64}$

As the areal capacitance and sheet resistance are related to the film thickness via $C_{A}=C_{V} t$ and $R_{s}=\left(\sigma_{D C} t\right)^{-1}$, it is possible to write Equation 10 in an alternative form:

$$
\tau=\frac{C_{V}}{\sigma_{D C}} L^{2}+\frac{C_{V}}{G_{A}} t
$$

This form is of interest as it depends on intrinsic and dimensional parameters only and includes the dependence on electrode thickness.

We can also apply this model to the data for $C / A$ as a function of $L$. To do this we combine Equations 9 and 10 to give:

$$
\frac{C}{A}=C_{A}\left[1-\frac{\dot{V}}{\Delta V}\left(C_{A} R_{S} L^{2}+\frac{C_{A}}{G_{A}}\right)\left(1-\exp \left[\frac{-\Delta V / \dot{V}}{\left(C_{A} R_{S} L^{2}+C_{A} / G_{A}\right)}\right]\right)\right]
$$

As all parameters in this expression are known $\left(C_{A}, R_{s}, G_{A}, \Delta V\right)$ we can plot Equation 12 as a function of $L$ for different $\dot{V}$ and compare with the experimental data presented in Figure 3C. Although we find good agreement at low rates, the fit is noticeably poorer for $\dot{V}$ $>1000 \mathrm{mV} / \mathrm{s}$. This isn't unexpected; at such high charge/discharge rates a series resistorcapacitor combination is no longer an appropriate representation of this electrode system. To provide evidence for this, we have performed a separate electrochemical impedance spectroscopy analysis of the behaviour of these electrode as a function of sample $L$, which has been included in the SI. This analysis demonstrates that under rapid charge discharge conditions (in this case, a high frequency voltage perturbation) a Warburg-like electrode response is observed indicating that the electrode behaviour becomes limited by electrical 
transport to the back of the film (SI Figure S6). As such, a more sophisticated model for this electrode system consists of a single-arm transmission line with a distributed capacitance that may be accessed via an incremental resistance through the plane of the film (SI Figure S7). However, we feel that the series resistor-capacitor circuit model that has been applied to CV electrode behaviour provides a simple and insightful treatment of lateral dimension effects that is applicable over a wide range of charge discharge rates relevant for SC operation.

In addition to the sample length dependence of capacitive charge storage, for completeness we have also performed the same CV analysis for the converse situation; with the sample length kept constant $(L=0.5 \mathrm{~cm})$, while varying the width $(W=0.8 \mathrm{~cm}$ to $4.5 \mathrm{~cm})$. As shown in Figure 4E and F, the $W$ dependence is straightforward. Although $C / A$ falls with scan rate in line with Equation 9, it is invariant with sample width, $W$, at all rates. This is as expected because the sample width does not appear in Equation 12.

This simple CV analysis demonstrates the influence of film lateral dimensions on the charging and discharging time constant, which in turn causes $C / A$ to be diminished for longer films. Although fairly intuitive, this behaviour has not been identified or studied in detail previously and we feel these insights will be very important for future transparent SC design. 
As indicated by Figure 4A and C, $C / A$ begins to fall once either the scan rate or sample length are increase above a certain value. The design of real large-area supercapacitor electrodes would be facilitated by an understanding of the relationship between sample length and scan rate when the effective capacitance begins to deviate from the rate-independent value. The model above can be used to provide this relationship.

The $C / A$ as obtained from Equation 9 is essentially constant for a low values of $\dot{V} \tau / \Delta V$ . By analysis of Equation 9 (i.e. by expanding the exponential and equating the zeroth order, low rate approximation with the second order high rate approximation), it can be shown that the $C / A$ begins to fall off approximately when $\dot{V} \tau / \Delta V=0.5$. Using Equation 11 this means that for a given sample length the maximum scan rate before the $C / A$ begins to fall off is given by:

$\dot{V}_{M a x}=\frac{\Delta V / 2}{\left(C_{V} L^{2} / \sigma_{D C}+C_{V} t / G_{A}\right)}$

Or alternatively the maximum sample length for effective performance at a given scan rate:

$$
L_{M a x}=\sqrt{\frac{\sigma_{D C}}{C_{V}}\left(\frac{\Delta V / 2}{\dot{V}}-\frac{C_{V} t}{G_{A}}\right)}
$$

We note that these expressions can be expressed alternatively as a function of transmittance rather than thickness using Equation 5. However, for the sake of simplicity we leave them in the current form.

These expressions show that as the electrode length is increased, the maximum rate at which energy can be extracted, as expressed by $\dot{V}_{\text {Max }}$, falls rapidly. Attempts to extract charge at a higher rate will result in a fall-off in effective capacitance (and so accessible energy). To 
demonstrate, in Figure 5 we use Equation 13 to plot the maximum achievable scan rate as a function of $L$ using the parameters established above for PEDOT:PSS, and the electrolyte used here: $\sigma_{D C}=936 \mathrm{~S} / \mathrm{cm}, C_{V}=41 \mathrm{~F} / \mathrm{cm}^{3}, \Delta V=0.6 \mathrm{~V}, G_{A}=18 \mathrm{~S} / \mathrm{m}^{2}$. These graphs clearly demonstrate the limitations on the performance transparent SC electrodes in terms of size or charge and discharge rate. For example, if the electrode transparency is set at $90 \%$ (effectively setting the thickness) and a maximum scan rate of $1 \mathrm{~V} / \mathrm{s}$ is required, the sample length can be no more than $1 \mathrm{~cm}$ before the $C / A$ start falling off from its rate-independent value.

\section{Large area device measurements}

Presently, the largest transparent SC device described in the literature ${ }^{26}$ is only $4.5 \mathrm{~cm}^{2}$. Given that applications will no doubt require larger area devices, capable of greater absolute charge storage, it is necessary to demonstrate what kind of performance is possible. The spray system used in this work is capable of producing films with dimensions of $18 \mathrm{~cm}$ by $18 \mathrm{~cm}$, potentially giving a device area of $324 \mathrm{~cm}^{2}$. However, was shown in the previous section, the absolute capacitance of devices with an $L$ dimension greater than $\sim 5 \mathrm{~cm}$ aren't able to store significantly more charge at the charge/discharge rates of interest for SC applications $(\sim 100 \mathrm{~s}$ of $\mathrm{mV} / \mathrm{s})$. Accordingly, here we present measurements for devices with dimensions of $W=18$ $\mathrm{cm}$ and $L \leq 6 \mathrm{~cm}$ ), giving a maximum electrode footprint of $108 \mathrm{~cm}^{2}$. A pair of films produced from a single $18 \mathrm{~cm}$ square piece is shown in Figure $6 \mathrm{~A}$ with film transmittance of $95 \%$. These electrodes are substantially larger than those used in any transparent SC device published previously.

Using a two electrode configuration, these electrodes were tested in a large beaker by cyclic voltammetry at a variety of scan rates (50 to $1500 \mathrm{mV} / \mathrm{s}$ ). Shown in Figure 6B are CVs expressed as differential capacitance $((d q / d V) / A)$ for four device footprints $\left(\mathrm{A}=18 \mathrm{~cm}^{2}\right.$ to $108 \mathrm{~cm}^{2}$ ) at $50 \mathrm{mV} / \mathrm{s}$. Measured areal capacitances were found by integrating over the potential 
window and are plotted $v s$. device length for a range of scan rates in Figure 6C. In all cases, $C / A$ falls with sample length, although this phenomenon is less severe for a low scan rates as was observed from single electrode measurements. Shown in Figure $6 \mathrm{D}$ is the absolute capacitance (i.e. $C=L W \times(C / A)$ ) plotted vs. electrode length. For the largest area device, we measured $12.5 \mathrm{mF}$ at $50 \mathrm{mV} / \mathrm{s}$. It is clear from this data that the absolute capacitance increases at best sublinearly with length over the size range. This is entirely consistent with the results of Figure 5 and show that for combinations of long lengths and high rates increasing the electrode length gives fast diminishing returns. We show this more clearly in Figure $6 \mathrm{E}$ by plotting the absolute capacitance as a function of rate for two electrode lengths, $L=1$ and $6 \mathrm{~cm}$. It is clear from this graph that while at low rates, a six-fold increase in electrode length results in a doubling of capacitance, at high rates, no benefit is seen from the electrode length increase.

As shown above, our largest area device gave $\mathrm{C}=12.5 \mathrm{mF}$ at $50 \mathrm{mV} / \mathrm{s}$ for a total transmittance of $90 \%$. In comparison, Jung et al. reported a transparent SC device with $A=4.5$ $\mathrm{cm}^{2}$ composed of electrodes with $T=71 \%$ at $500 \mathrm{~nm}$, which gave a capacitance of $2.1 \mathrm{mF}$ by constant current charge discharge measurements $\left(j=5 \mu \mathrm{A} / \mathrm{cm}^{2}\right)$. Although this value is 5 times less than reported here, care must be taken when making comparison. This is because the two devices possess different aspect ratio, different transmittance and were characterised by different electrochemical techniques. 


\section{CONCLUSIONS}

This work furthers our understanding of the properties of transparent SC electrodes by characterising PEDOT:PSS ultra-thin electrodes in terms of three key materials properties; the optical conductivity, $\sigma_{o p}$, the DC electrical conductivity, $\sigma_{D C}$ and the volumetric capacitance, $C_{V}$. Together, these determine the performance of a given transparent supercapacitor material, which may be summarised as an electrical and capacitive figure of merit for transparent supercapacitors; $\mathrm{FoM}_{\mathrm{e}}=\sigma_{D C} / \sigma_{o p}$, and $\mathrm{FoM}_{\mathrm{c}}=C_{V} / \sigma_{o p}$. Use of these metrics will facilitate rigorous comparison of candidate material systems for application as transparent SC electrodes, necessary for continued development of this field. The use of a spray coating deposition system combined with a secondary doping treatment using formic acid enabled the production high quality PEDOT:PSS films without percolation effects even for very high transmittance $(T=99 \%)$.

This work also identifies lateral dimension effects which cause the measured areal capacitance to be lower than expected when scaling up the electrode area (i.e. A $>1 \mathrm{~cm}^{2}$ ). These effects occur due to the need for current collection through the plane of the film and have implications for the capacitance that is available when using electrodes with a given lateral size when charged/discharged at a given rate. Accordingly, to assist with transparent supercapacitor design, we have developed simple equations that take these effects into account, enabling the analysis of data for capacitance versus rate or electrode dimensions. This behaviour will predominate situations where the thin film material acts as the charge storage material and current collector simultaneously, and as such highly relevant for printed electronics applications. 


\section{METHODS}

\section{Film Preparation}

An aqueous dispersion of PEDOT:PSS was obtained from Heraeus (Clevios PH 1000) and diluted with water to a concentration of $0.75 \mathrm{mg} / \mathrm{mL}$. This was then used to produce PEDOT:PSS films on polyethlyene terephthalate (PET) substrates using the method described in McCarthy et al. ${ }^{53}$ In brief, the diluted polymer solution was loaded into a graduated reservoir and attached to a Harddner and Steenbeck Infinity airbrush spray system. Control over the airbrush was provided by a Janome JR2300N robot. The PET substrates were cleaned with surfactant solution and isopropanol prior to use and placed on the robot substrate stage, heated to $110^{\circ} \mathrm{C}$. Further details regarding the spraying parameters employed can be found in the SI.

The film lateral dimensions were controlled by the spraying program, limited by the dimensions of the substrate state (here $18 \mathrm{~cm}$ by $18 \mathrm{~cm}$ ). The thickness of the films was controlled by the number of repeated passes made by the spray, which delivers a greater total volume of polymer solution onto a given area of the substrate. Films were subsequently characterised as-prepared or subject to a formic acid (FA) post treatment. This involved submerging the PEDOT:PSS films into the FA for 5 seconds then allowing the films to dry in air.

\section{Optoelectrical Characterisation of Films}

Optical transmittance spectra of the PEDOT:PSS films were obtained in the visible range (400-800 nm) using a Cary 600i spectrophotometer. In all cases the PET background absorbance was subtracted. Film thickness was determined by contact profilometry (Dektak 6M, Veeco Instruments) for PEDOT:PSS films sprayed separately onto glass substrates. Here film thickness values were taken as the mean thickness obtained by four height profiles 
obtained for different regions of the film. These measurements were performed after exposure to FA as such secondary doping treatments are known to alter the film density. ${ }^{53}$ The four-point probe technique was used to characterise the DC electrical properties of the films. To do so, electrodes contacts were created on the film surface using an alcohol based conductive silver paint (Agar Scientific). The electrode separation and width were $\sim 1 \mathrm{~cm}$ and $\sim 0.5 \mathrm{~cm}$, respectively. Current-voltage curves were obtained using a Keithley 2400 source meter.

\section{Electrochemical Characterisation of Films}

Electrochemical testing was carried out in a three electrode cell consisting of a carbon counter electrode and $\mathrm{Ag} / \mathrm{AgCl}$ reference. The electrolyte used was $0.5 \mathrm{M} \mathrm{K}_{2} \mathrm{SO}_{4}(\mathrm{aq})$. PEDOT:PSS working electrodes were formed by cutting strips from the main film and confining the electrolyte exposed area using adhesive tape. Silver paint was again used to form contacts to the external circuit. The electrolyte exposed area was varied during different experiments. Here we have define the electrode width $(W)$ to be the dimension parallel to the electrolyte solution and the length $(L)$ the perpendicular dimension (i.e. greater $L$ are more distant from the contact). The specific dimensions used for various measurements are indicated in the results section. For the $L$ and $W$ dependence measurements a large electrode was prepared, which was sequentially cut to the required smaller dimensions after the necessary measurements for a given size were completed.

Electrochemical testing using the three electrode cell consisted of cyclic voltammetry, and electrochemical impedance spectroscopy. CVs were obtained between the potential limits of 0.1 to $0.7 \mathrm{~V} v s$. the $\mathrm{Ag} / \mathrm{AgCl}$ reference couple. Scan rates were varied from 100 to 5000 $\mathrm{mV} / \mathrm{s}$. Potentiostatic EIS was carried out at a DC bias of $0.4 \mathrm{~V}$ vs. $\mathrm{Ag} / \mathrm{AgCl}$ using a $10 \mathrm{mV} \mathrm{AC}$ perturbation varying between $0.1 \mathrm{~Hz}$ and $100 \mathrm{kHz}$. Immediately prior to acquiring the EIS spectra working electrodes were conditioned at the as-mentioned DC bias for 3 minutes. This 
DC bias was chosen as the mid-potential used for the CV measurements. To determine a value for the density of FA-treated PEDOT:PSS a free-standing film was prepared by evaporative casting into a teflon mould and measuring both its mass and dimensions.

Symmetric two electrode measurements were performed in a large beaker containing $0.5 \mathrm{M} \mathrm{K}_{2} \mathrm{SO}_{4}(\mathrm{aq})$ electrolyte. For these measurements two films of equal dimensions and transmittance were positioned $1 \mathrm{~mm}$ apart. Here, CVs were performed using films with different areas. This was achieved by varying the film length $(L \leq 6 \mathrm{~cm})$ while holding the width constant $(W=18 \mathrm{~cm})$, giving a maximum film area of $108 \mathrm{~cm}^{2}$.

\section{SUPPORTING INFORMATION}

This includes (1) details of the airbrush system parameters, (2) supporting figures regarding the effect of FA treatment on the PEDOT:PSS transmittance spectra and absence of percolation effects for ultra thin films, (3) method preparation of Iljin SWNT dispersions, (4) a reproduction of Figure $2 \mathrm{D}$ to indicate the authors of the included literature data, (5) an electrochemical impedance spectroscopy analysis of the $\mathrm{L}$ dependence of electrode AC behaviour. This material is available free of charge via the Internet at http://pubs.acs.org.

\section{ACKNOWLEDGEMENTS}

The authors would like to thank Dr. Joseph McCarthy and Mr. Richard Coull for discussions and assistance with use of the robotic spraying system. We have received support from the Science Foundation Ireland (SFI) funded centre AMBER (SFI/12/RC/2278). In addition, JNC acknowledges the European Research Council (SEMANTICS) and SFI (11/PI/1087) for financial support. 


\section{FIGURES}
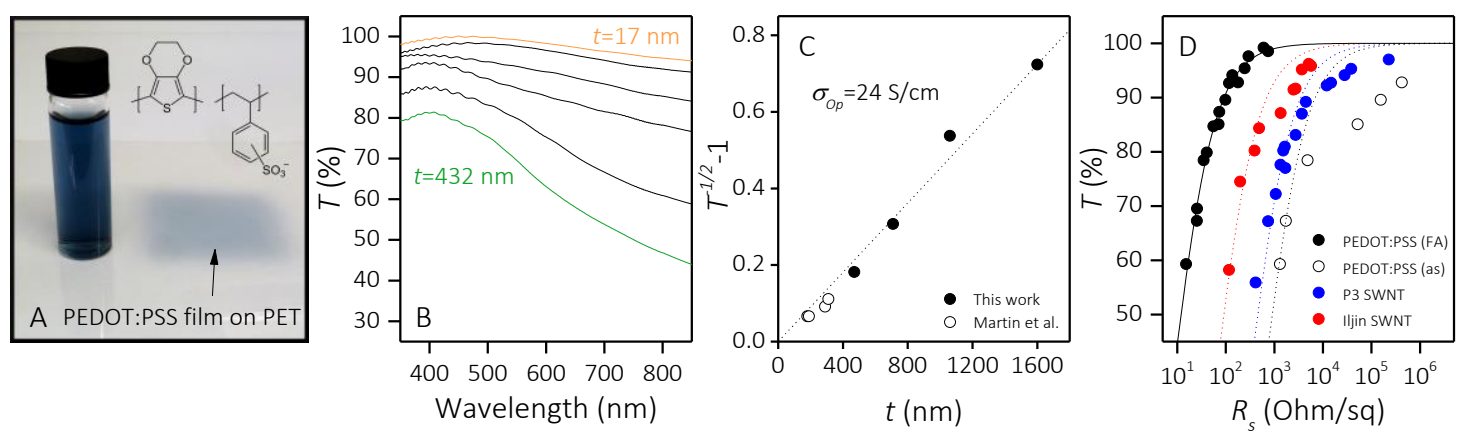

Figure 1: Characterisation of the optoelectronic properties of sprayed PEDOT:PSS films. (A) Photograph of an aqueous PEDOT:PSS dispersion and a film $(5 \mathrm{~cm}$ by $5 \mathrm{~cm}, T=70 \%)$ on a PET plastic substrate. (B) UV-visible transmittance spectra of various sprayed PEDOT:PSS films. (C) Plot of $T^{-1 / 2}-1$ (at $500 \mathrm{~nm}$ ) vs. film thickness, $t$, to determine the optical conductivity of PEDOT:PSS using Equation 5. Unfilled points are spin-coated PEDOT:PSS films described by Martin et ll $^{43}$ (D) Film transmittance at $550 \mathrm{~nm}$ as a function of sheet resistance for the asprepared PEDOT:PSS films (unfilled black circles), after treatment with FA (filled black circles) and data for disordered SWNT networks (P3 SWNTs and Iljin SWNTs). Fits to these data use Equation 4. 

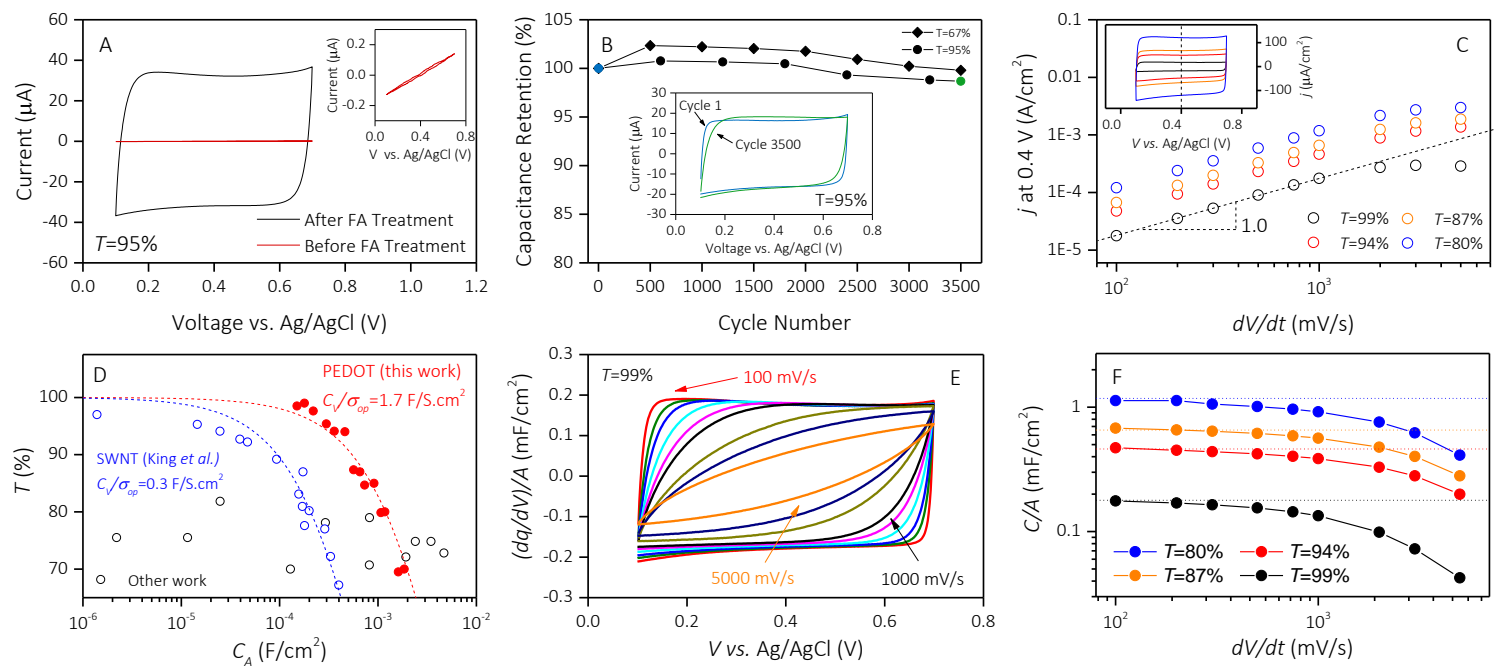

Figure 2: Characterisation of the charge storage properties of small area PEDOT:PSS films ( $L=1 \mathrm{~cm}$ and $W=1 \mathrm{~cm}$ ) by cyclic voltammetry. (A) CV response before (red) and after (black) FA treatment. B) Capacitance retention of FA-treated films upon repeated cycling. Inset compares the $\mathrm{CV}$ response at cycle 1 and 3500 for a film with $\mathrm{T}=95 \%$. (C) Current density evaluated from $\mathrm{CVs}$ at $0.4 \mathrm{~V}$ vs. $\mathrm{Ag} / \mathrm{AgCl}$ for FA-treated samples at various scan rates. Ordinate intercepts enable extraction of the rate-independent areal capacitance, $C_{A}$. The $(\mathrm{C})$ inset gives example $\mathrm{CV}$ s for four samples $(d V / d t=100 \mathrm{mV} / \mathrm{s})$. (D) $C_{A}$ plotted as a function of $T$, which includes a range of data are taken from the literature (SI Figure S4 contains a reproduction of this figure indicating sources of the literature data, not shown here for clarity). Dashed lines are fits to Equation 3 with $C_{V} / \sigma_{o p}=1.7 \mathrm{~F} / \mathrm{S} . \mathrm{cm}^{2}$. (E) CVs for a single film $(T=99 \%)$ at various scan rates. Current density is reexpressed as a differential capacitance. (F) $C / A$ found by integrating $\mathrm{CV}$ current over the potential window. Dashed lines indicate the $C_{A}$ values obtained in (C). 

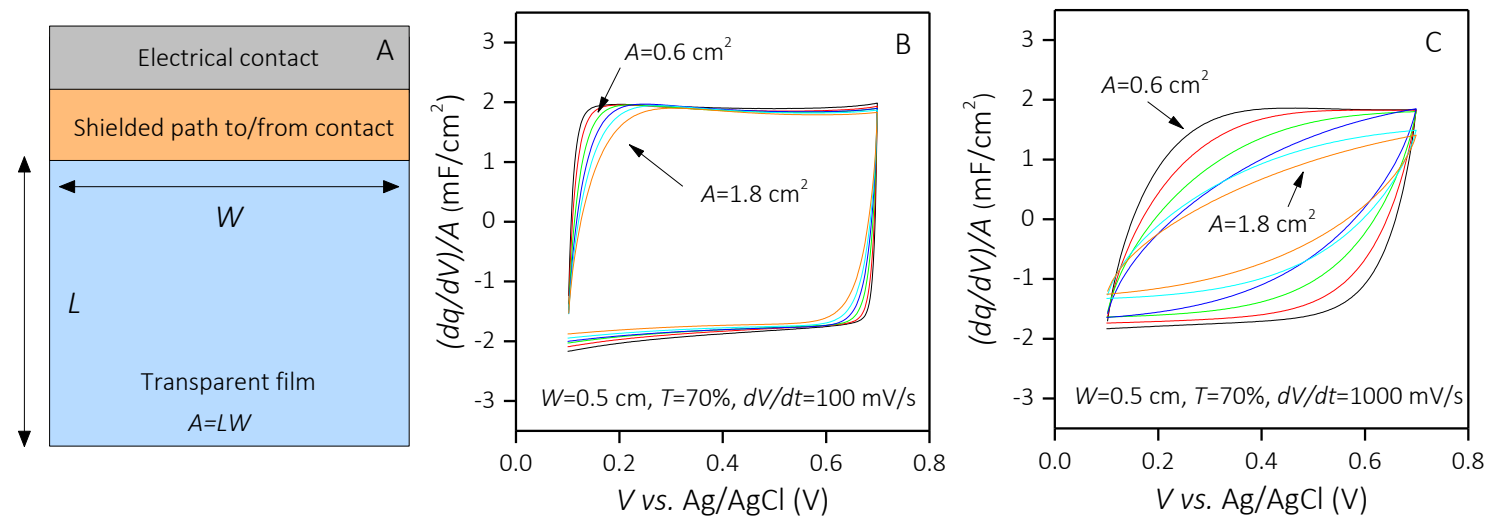

Figure 3: Effect of film lateral dimensions on CV behaviour of FA-treated PEDOT:PSS films. (A) Diagram of electrode indicating length $(L)$ and width $(W)$ dimensions with relation to the electrical contact (top edge). (B) CVs at $\dot{V}=100 \mathrm{mV} / \mathrm{s}$ and (C) $\dot{V}=1000 \mathrm{mV} / \mathrm{s}$ for a $T=70 \%$ film. Film area is varied from $A=0.6 \mathrm{~cm}^{2}$ to $1.8 \mathrm{~cm}^{2}$, by altering the length from $L=1.2 \mathrm{~cm}$ and $3.6 \mathrm{~cm}$. (width constant, $W=0.5 \mathrm{~cm}$ ). Current density is expressed as a differential capacitance. 

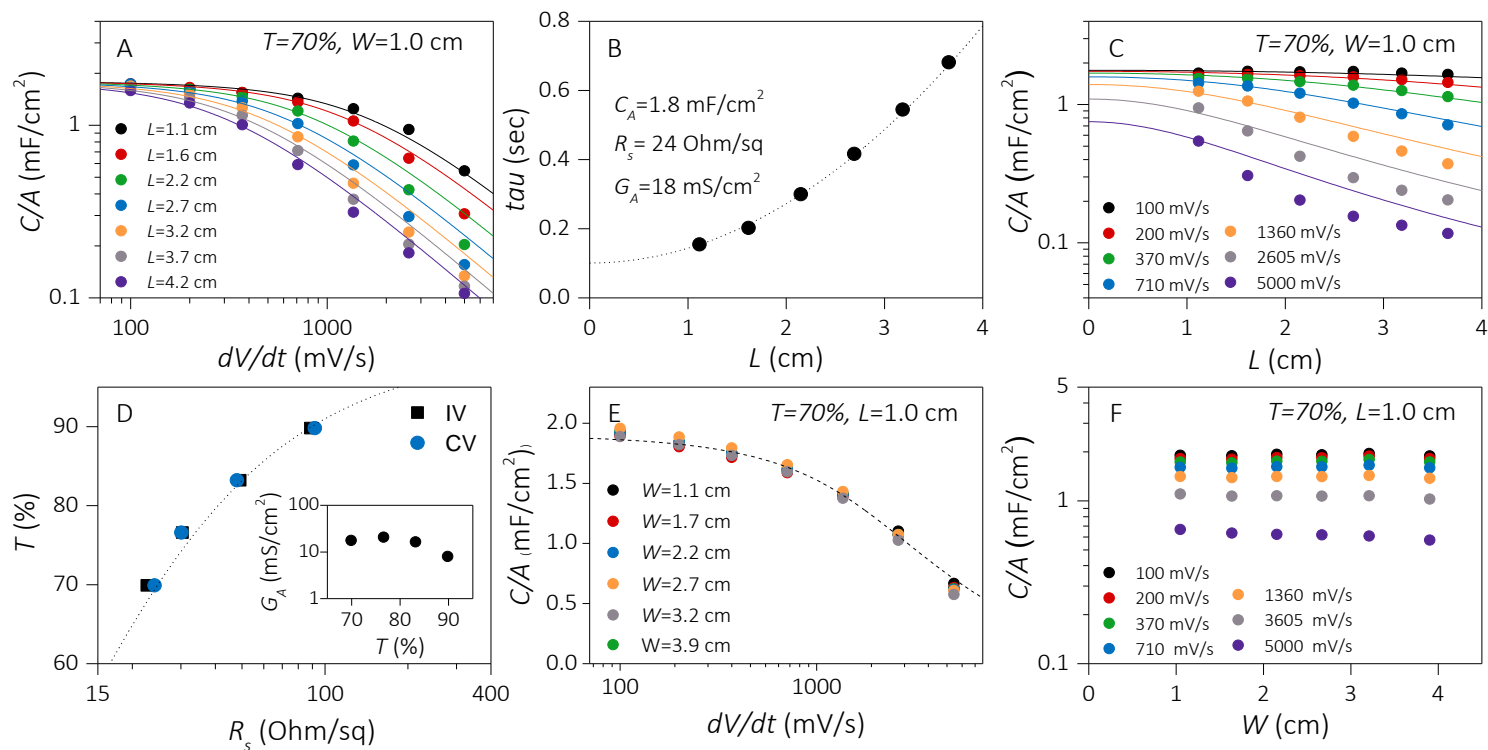

Figure 4: Size dependence of the charge storage properties of PEDOT:PSS films. (A) $C / A$ data obtained by integrating the current passed for CVs at various $\dot{V}$ for a film with different sample lengths ( $L=1.1$ to $4.2 \mathrm{~cm}$ ), keeping the width constant. Solid lines are fits of Equation 9 , providing the time constant, $\tau$, as a function of sample length, plotted in (B). Here the dashed line is a fit of Equation 10, with $C_{A}=1.8 \mathrm{mF} / \mathrm{cm}^{2}$ and $R_{s}=24 \mathrm{ohm} / \mathrm{sq}$ and $G_{A}=18 \mathrm{mS} / \mathrm{cm}^{2}$ for this film. (C) $C / A$ data plotted as a function of sample length for various $\dot{V}$. Using the parameters found in Figure 4B, Equation 12 has been plotted with this data. (D) $R_{s}$ values extracted from the $L$ dependence analysis for various films, compared with those obtained by IV measurements. Inset shows electrolyte conductance values obtained for these films. (E) $C / A$ obtained similarly as for (A), although here the film width is varied ( $W=1.1$ to $3.9 \mathrm{~cm}$ ), keeping the length constant. (F) $C / A$ plotted as a function of sample width at various $\dot{V}$. 


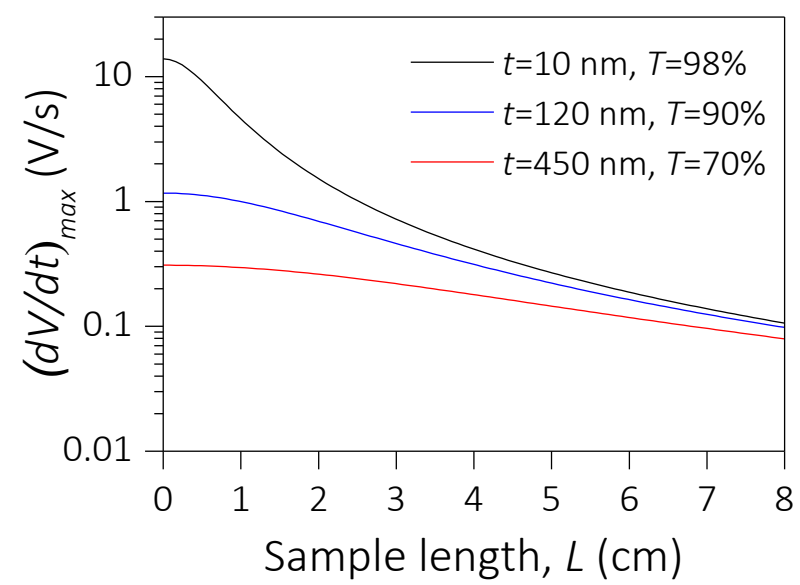

Figure 5: Scan rate above which the $\mathrm{CV}$ response deviates from the $C_{A}$ as a function of sample length, $L$. The three traces show the dependence on film transmittance (and thickness) for three different films. 


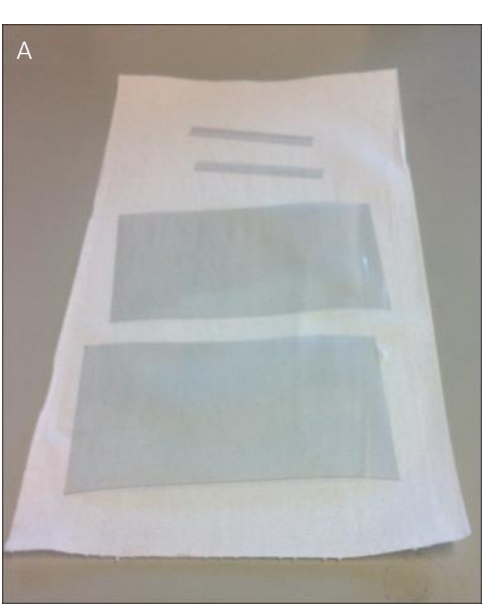

$W=18 \mathrm{~cm}, L=9 \mathrm{~cm} T=95 \%$
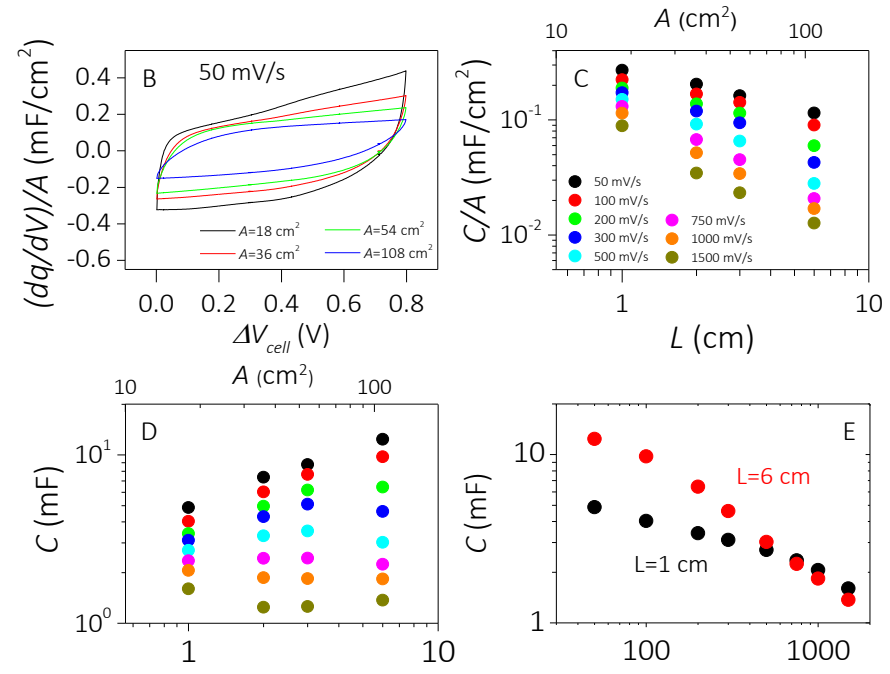

$L(\mathrm{~cm})$

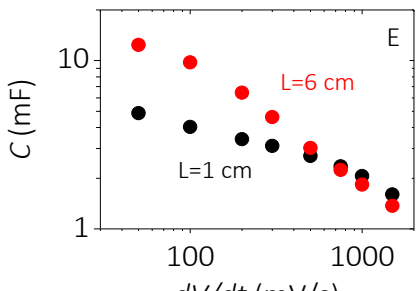

$d V / d t(m V / s)$

Figure 6: Two electrode charge storage measurements for various transparent SCs $\left(T_{\text {single }}=95 \%\right)$. Electrode width was kept constant $(W=18 \mathrm{~cm})$ and length varied $(L=1 \mathrm{~cm}$ to 6 $\mathrm{cm})$ to produce device areas from $A=18 \mathrm{~cm}^{2}$ to $108 \mathrm{~cm}^{2}$. (A) Photo of two large-area sprayed PEDOT:PSS films $(18 \mathrm{~cm}$ by $9 \mathrm{~cm}$ ). (B) $\mathrm{CVs}$ obtained at $50 \mathrm{mV} / \mathrm{s}$ for the different device areas, expressed as a differential capacitance. (C) $C / A$ as a function of device length, found by integration of CVs at various scan rates $(d V / d t=50$ to $1500 \mathrm{mV} / \mathrm{s})$. (D) Absolute capacitance as a function of device length at various scan rates. Max. capacitance for largest device was 12.5 $\mathrm{mF}(d V / d t=50 \mathrm{mV} / \mathrm{s})$. (E) Absolute capacitance as a function of scan rate for $L=1$ and $6 \mathrm{~cm}$ (corresponding to $\mathrm{A}=18$ and $108 \mathrm{~cm}^{2}$ ). 


\section{REFERENCES}

1. $\quad$ Sirringhaus, H.; Kawase, T.; Friend, R. H.; Shimoda, T.; Inbasekaran, M.; Wu, W.; Woo, E. P., High-Resolution Inkjet Printing of All-Polymer Transistor Circuits. Science 2000, 290 (5499), 2123-2126.

2. $\quad$ Park, J.-U.; Hardy, M.; Kang, S. J.; Barton, K.; Adair, K.; Mukhopadhyay, D. k.; Lee, C. Y.; Strano, M. S.; Alleyne, A. G.; Georgiadis, J. G., et al., High-resolution electrohydrodynamic jet printing. Nat. Mater. 2007, 6 (10), 782-789.

3. Torrisi, F.; Hasan, T.; Wu, W.; Sun, Z.; Lombardo, A.; Kulmala, T. S.; Hsieh, G.-W.; Jung, S.; Bonaccorso, F.; Paul, P. J., et al., Inkjet-Printed Graphene Electronics. ACS Nano 2012, 6 (4), 2992-3006.

4. Ishikawa, F. N.; Chang, H.-k.; Ryu, K.; Chen, P.-c.; Badmaev, A.; Gomez De Arco, L.; Shen, G.; Zhou, C., Transparent Electronics Based on Transfer Printed Aligned Carbon Nanotubes on Rigid and Flexible Substrates. ACS Nano 2009, 3 (1), 73-79.

5. $\quad$ Lee, S.-K.; Kim, B. J.; Jang, H.; Yoon, S. C.; Lee, C.; Hong, B. H.; Rogers, J. A.; Cho, J. H.; Ahn, J.-H., Stretchable Graphene Transistors with Printed Dielectrics and Gate Electrodes. Nano Lett. 2011, 11 (11), 4642-4646.

6. $\quad$ Lee, S.; Lee, K.; Liu, C.-H.; Kulkarni, G. S.; Zhong, Z., Flexible and transparent all-graphene circuits for quaternary digital modulations. Nat. Commun. 2012, 3.

7. $\quad$ Wu, Z.; Chen, Z.; Du, X.; Logan, J. M.; Sippel, J.; Nikolou, M.; Kamaras, K.; Reynolds, J. R.; Tanner, D. B.; Hebard, A. F., et al., Transparent, Conductive Carbon Nanotube Films. Science 2004, 305 (5688), 1273-1276.

8. $\quad$ Sorel, S.; Khan, U.; Coleman, J. N., Flexible, transparent dielectric capacitors with nanostructured electrodes. Appl. Phys. Lett. 2012, 101 (10), 103106-103106-5.

9. Kudo, A.; Yanagi, H.; Ueda, K.; Hosono, H.; Kawazoe, H.; Yano, Y., Fabrication of transparent $\mathrm{p}-\mathrm{n}$ heterojunction thin film diodes based entirely on oxide semiconductors. Appl. Phys. Lett. 1999, 75 (18), 2851-2853.

10. Ju, S.; Facchetti, A.; Xuan, Y.; Liu, J.; Ishikawa, F.; Ye, P.; Zhou, C.; Marks, T. J.; Janes, D. B., Fabrication of fully transparent nanowire transistors for transparent and flexible electronics. Nat. Nanotechnol. 2007, 2 (6), 378-384.

11. Xiao, L.; Chen, Z.; Feng, C.; Liu, L.; Bai, Z.-Q.; Wang, Y.; Qian, L.; Zhang, Y.; Li, Q.; Jiang, K., et al., Flexible, Stretchable, Transparent Carbon Nanotube Thin Film Loudspeakers. Nano Lett. 2008, 8 (12), 4539-4545.

12. Nakamura, H.; Tohyama, K.; Tanaka, M.; Shinohara, S.; Tokunaga, Y.; Kurusu, F.; Koide, S.; Gotoh, M.; Karube, I., Development of a package-free transparent disposable biosensor chip for simultaneous measurements of blood constituents and investigation of its storage stability. Biosens. Bioelectron. 2007, 23 (5), 621-626.

13. Fan, F.-R.; Lin, L.; Zhu, G.; Wu, W.; Zhang, R.; Wang, Z. L., Transparent Triboelectric Nanogenerators and Self-Powered Pressure Sensors Based on Micropatterned Plastic Films. Nano Lett. 2012, 12 (6), 3109-3114.

14. Lunt, R. R.; Bulovic, V., Transparent, near-infrared organic photovoltaic solar cells for window and energy-scavenging applications. Appl. Phys. Lett. 2011, 98 (11), 113305.

15. Choi, D.; Choi, M.-Y.; Choi, W. M.; Shin, H.-J.; Park, H.-K.; Seo, J.-S.; Park, J.; Yoon, S.M.; Chae, S. J.; Lee, Y. H., et al., Fully Rollable Transparent Nanogenerators Based on Graphene Electrodes. Adv. Mater. 2010, 22 (19), 2187-2192.

16. Yang, Y.; Jeong, S.; Hu, L.; Wu, H.; Lee, S. W.; Cui, Y., Transparent lithium-ion batteries. Proc. Natl. Acad. Sci. U. S. A. 2011.

17. Chen, P.-C.; Shen, G.; Sukcharoenchoke, S.; Zhou, C., Flexible and transparent supercapacitor based on In2O3 nanowire/carbon nanotube heterogeneous films. Appl. Phys. Lett. 2009, 94 (4).

18. Invernale, M. A.; Seshadri, V.; Mamangun, D. M. D.; Ding, Y.; Filloramo, J.; Sotzing, G. A., Polythieno[3,4-b]thiophene as an Optically Transparent Ion-Storage Layer. Chem. Mater. 2009, 21 (14), 3332-3336. 
19. Sugimoto, W.; Yokoshima, K.; Ohuchi, K.; Murakami, Y.; Takasu, Y., Fabrication of Thinfilm, Flexible, and Transparent Electrodes Composed of Ruthenic Acid Nanosheets by Electrophoretic Deposition and Application to Electrochemical Capacitors. J. Electrochem. Soc. 2006, 153, A255-A260.

20. Moser, F.; Athouel, L.; Crosnier, O.; Favier, F.; Belanger, D.; Brousse, T., Transparent electrochemical capacitor based on electrodeposited $\mathrm{MnO} 2$ thin film electrodes and gel-type electrolyte. Electrochem. Commun. 2009, 11 (6), 1259-1261.

21. Yu, A.; Roes, I.; Davies, A.; Chen, Z., Ultrathin, transparent, and flexible graphene films for supercapacitor application. Appl. Phys. Lett. 2010, 96.

22. Ge, J.; Cheng, G.; Chen, L., Transparent and flexible electrodes and supercapacitors using polyaniline/single-walled carbon nanotube composite thin films. Nanoscale 2011, 3 (8), 3084-3088. 23. Hu, Y.; Zhu, H.; Wang, J.; Chen, Z., Synthesis of layered birnessite-type manganese oxide thin films on plastic substrates by chemical bath deposition for flexible transparent supercapacitors. $J$. Alloys Compd. 2011, 509 (42), 10234-10240.

24. Nakayama, M.; Okamura, K.; AthouÃ «l, L.; Crosnier, O.; Brousse, T., Fabrication of a Transparent Supercapacitor Electrode Consisting of Mn-Mo Oxide/CNT Nanocomposite. ECS Trans. 2012, 41 (22), 53-64.

25. Ryu, I.; Yang, M.; Kwon, H.; Park, H. K.; Do, Y. R.; Lee, S. B.; Yim, S., Coaxial RuO2-ITO Nanopillars for Transparent Supercapacitor Application. Langmuir 2014, 30 (6), 1704-1709.

26. Jung, H. Y.; Karimi, M. B.; Hahm, M. G.; Ajayan, P. M.; Jung, Y. J., Transparent, flexible supercapacitors from nano-engineered carbon films. Sci. Rep. 2012, 2.

27. King, P. J.; Higgins, T. M.; De, S.; Nicoloso, N.; Coleman, J. N., Percolation Effects in Supercapacitors with Thin, Transparent Carbon Nanotube Electrodes. ACS Nano 2012.

28. $\quad$ Gao, K.; Shao, Z.; Wu, X.; Wang, X.; Zhang, Y.; Wang, W.; Wang, F., Paper-based transparent flexible thin film supercapacitors. Nanoscale 2013, 5 (12), 5307.

29. Gao, Y.; Zhou, Y. S.; Xiong, W.; Jiang, L. J.; Mahjouri-samani, M.; Thirugnanam, P.; Huang, X.; Wang, M. M.; Jiang, L.; Lu, Y. F., Transparent, flexible, and solid-state supercapacitors based on graphene electrodes. APL Mater. 2013, 1 (1).

30. Lin, H.; Li, L.; Ren, J.; Cai, Z.; Qiu, L.; Yang, Z.; Peng, H., Conducting polymer composite film incorporated with aligned carbon nanotubes for transparent, flexible and efficient supercapacitor. Sci. Rep. 2013, 3, 1353.

31. Nam, I.; Park, S.; Kim, G.-P.; Park, J.; Yi, J., Transparent and ultra-bendable all-solid-state supercapacitors without percolation problems. Chem. Sci. 2013, 4 (4), 1663-1667.

32. Niu, Z.; Zhou, W.; Chen, J.; Feng, G.; Li, H.; Hu, Y.; Ma, W.; Dong, H.; Li, J.; Xie, S., A Repeated Halving Approach to Fabricate Ultrathin Single-Walled Carbon Nanotube Films for Transparent Supercapacitors. Small 2013, 9 (4), 518-524.

33. Chen, T.; Peng, H.; Durstock, M.; Dai, L., High-performance transparent and stretchable allsolid supercapacitors based on highly aligned carbon nanotube sheets. Sci. Rep. 2014, 4.

34. Chen, T.; Xue, Y.; Roy, A. K.; Dai, L., Transparent and Stretchable High-Performance Supercapacitors Based on Wrinkled Graphene Electrodes. ACS Nano 2014, 8 (1), 1039-1046.

35. Fan, X.; Chen, T.; Dai, L., Graphene networks for high-performance flexible and transparent supercapacitors. RSC Adv. 2014, 4 (70), 36996-37002.

36. Kim, Y. H.; Sachse, C.; Machala, M. L.; May, C.; Müller-Meskamp, L.; Leo, K., Highly Conductive PEDOT:PSS Electrode with Optimized Solvent and Thermal Post-Treatment for ITOFree Organic Solar Cells. Adv. Funct. Mater. 2011, 21 (6), 1076-1081.

37. De, S.; Coleman, J. N., The effects of percolation in nanostructured transparent conductors. MRS Bull. 2011, 36 (10), 774-781.

38. Ahlswede, E.; Mühleisen, W.; bin Moh Wahi, M. W.; Hanisch, J.; Powalla, M., Highly efficient organic solar cells with printable low-cost transparent contacts. Appl. Phys. Lett. 2008, 92 (14), 143307.

39. Tait, J. G.; Worfolk, B. J.; Maloney, S. A.; Hauger, T. C.; Elias, A. L.; Buriak, J. M.; Harris, K. D., Spray coated high-conductivity PEDOT:PSS transparent electrodes for stretchable and mechanically-robust organic solar cells. Sol. Energy Mater. Sol. Cells 2013, 110 (0), 98-106. 
40. Elschner, A.; Kirchmeyer, S.; Lovenich, W.; Merker, U.; Reuter, K., PEDOT: Principles and Applications of an Intrinsically Conductive Polymer. 1 edition ed.; CRC Press: Boca Raton, FL, 2010; p 377.

41. Carlberg, J. C.; Inganäs, O., Poly(3,4-ethylenedioxythiophene) as Electrode Material in Electrochemical Capacitors. J. Electrochem. Soc. 1997, 144 (4), L61-L64.

42. $\quad$ Dressel, M.; Gruner, G., Electrodynamics of Solids: Optical Properties of Electrons in Matter. Cambridge University Press: 2002; p 490.

43. Martin, B. D.; Nikolov, N.; Pollack, S. K.; Saprigin, A.; Shashidhar, R.; Zhang, F.; Heiney, P. A., Hydroxylated secondary dopants for surface resistance enhancement in transparent poly $(3,4-$ ethylenedioxythiophene)-poly(styrenesulfonate) thin films. Synth. Met. 2004, 142 (1-3), 187-193. 44. De, S.; Higgins, T. M.; Lyons, P. E.; Doherty, E. M.; Nirmalraj, P. N.; Blau, W. J.; Boland, J. J.; Coleman, J. N., Silver Nanowire Networks as Flexible, Transparent, Conducting Films: Extremely High DC to Optical Conductivity Ratios. ACS Nano 2009, 3 (7), 1767-1774.

45. Scardaci, V.; Coull, R.; Lyons, P. E.; Rickard, D.; Coleman, J. N., Spray deposition of highly transparent, low-resistance networks of silver nanowires over large areas. Small 2011, 7 (18), 26212628.

46. Ruzicka, B.; Degiorgi, L.; Gaal, R.; Thien-Nga, L.; Bacsa, R.; Salvetat, J. P.; Forro, L., Optical and dc conductivity study of potassium-doped single-walled carbon nanotube films. Phys. Rev. B: Condens. Matter 2000, 61 (4), R2468-R2471.

47. Doherty, E. M.; De, S.; Lyons, P. E.; Shmeliov, A.; Nirmalraj, P. N.; Scardaci, V.; Joimel, J.; Blau, W. J.; Boland, J. J.; Coleman, J. N., The spatial uniformity and electromechanical stability of transparent, conductive films of single walled nanotubes. Carbon 2009, 47 (10), 2466-2473.

48. De, S.; King, P. J.; Lotya, M.; O'Neill, A.; Doherty, E. M.; Hernandez, Y.; Duesberg, G. S.; Coleman, J. N., Flexible, Transparent, Conducting Films of Randomly Stacked Graphene from Surfactant-Stabilized, Oxide-Free Graphene Dispersions. Small 2010, 6, 458-464.

49. Higgins, T. M.; McAteer, D.; Coelho, J. C. M.; Sanchez, B. M.; Gholamvand, Z.; Moriarty, G.; McEvoy, N.; Berner, N. C.; Duesberg, G. S.; Nicolosi, V., et al., Effect of percolation on the capacitance of supercapacitor electrodes prepared from composites of manganese dioxide nanoplatelets and carbon nanotubes. ACS Nano 2014, 8 (9), 9567-9579.

50. Nardes, A. M.; Kemerink, M.; de Kok, M. M.; Vinken, E.; Maturova, K.; Janssen, R. A. J., Conductivity, work function, and environmental stability of PEDOT:PSS thin films treated with sorbitol. Org. Electron. 2008, 9 (5), 727-734.

51. Alemu, D.; Wei, H.-Y.; Ho, K.-C.; Chu, C.-W., Highly conductive PEDOT:PSS electrode by simple film treatment with methanol for ITO-free polymer solar cells. Energy Environ. Sci. 2012, 5 (11), 9662-9671.

52. Xia, Y.; Sun, K.; Ouyang, J., Solution-Processed Metallic Conducting Polymer Films as Transparent Electrode of Optoelectronic Devices. Adv. Mater. 2012, 24 (18), 2436-2440.

53. McCarthy, J. E.; Hanley, C. A.; Brennan, L. J.; Lambertini, V. G.; Gun'ko, Y. K., Fabrication of highly transparent and conducting PEDOT:PSS films using a formic acid treatment. J. Mater. Chem. C 2013, 2 (4), 764-770.

54. De, S.; Coleman, J. N., Are There Fundamental Limitations on the Sheet Resistance and Transmittance of Thin Graphene Films? ACS Nano 2010, 4 (5), 2713.

55. De, S.; King, P. J.; Lyons, P. E.; Khan, U.; Coleman, J. N., Size Effects and the Problem with Percolation in Nanostructured Transparent Conductors. ACS Nano 2010, 4, 7064-7072.

56. Nardes, A. M.; Kemerink, M.; Janssen, R. A. J., Anisotropic hopping conduction in spincoated PEDOT:PSS thin films. Physical Review B 2007, 76 (8), 085208.

57. Bard, A. J.; Faulkner, L. R., Electrochemical Methods: Fundamentals and Applications. 2 ed.; Wiley: 2000.

58. Heywang, G.; Jonas, F., Poly(alkylenedioxythiophene)s—new, very stable conducting polymers. Adv. Mater. 1992, 4 (2), 116-118.

59. Augustyn, V.; Come, J.; Lowe, M. A.; Kim, J. W.; Taberna, P.-L.; Tolbert, S. H.; Abruña, H. D.; Simon, P.; Dunn, B., High-rate electrochemical energy storage through Li+ intercalation pseudocapacitance. Nat. Mater. 2013, 12 (6), 518-522. 
60. Largeot, C.; Portet, C.; Chmiola, J.; Taberna, P.-L.; Gogotsi, Y.; Simon, P., Relation between the Ion Size and Pore Size for an Electric Double-Layer Capacitor. J. Am. Chem. Soc. 2008, 130 (9), 2730-2731.

61. Lang, X.; Hirata, A.; Fujita, T.; Chen, M., Nanoporous metal/oxide hybrid electrodes for electrochemical supercapacitors. Nat. Nanotechnol. 2011, 6 (4), 232-236.

62. Toupin, M.; Brousse, T.; Belanger, D., Charge Storage Mechanism of MnO2 Electrode Used in Aqueous Electrochemical Capacitor. Chem. Mater. 2004, 16, 3184-3190.

63. Stoller, M. D.; Ruoff, R. S., Best Practice Methods for Determining an Electrode Material's Performance for Ultracapacitors. Energy Environ. Sci. 2010, 3, 1294-1301.

64. Lide, D. R., CRC Handbook of Chemistry and Physics, 89th Edition. Taylor \& Francis: 2008; p 2736. 